\title{
Pavlovian conditioning with ingested saline solution as the US
}

\author{
JOHN M. KRUSE and VINCENT M. LOLORDO \\ Dalhousie University, Halifax, Nova Scotia, Canada
}

\begin{abstract}
Experience with $2 \%$ saline as the sole available fluid for drinking produced a decrement in preference for $.9 \%$ saline in a two-bottle test with water as the alternative. This outcome was interpreted as a consequence of Pavlovian conditioning, with the taste of salt acting as the conditioned stimulus and postingestive consequences of saline ingestion acting as the unconditioned stimulus. An overshadowing group experienced salt mixed with vanilla as its sole fluid during the conditioning phase. This group showed an attenuated saline aversion relative to a group that drank $2 \%$ saline solution during the conditioning phase. In addition, a vanilla aversion developed in the former group. In the second experiment, conditioning of the aversion to the taste of vanilla mixed with $2 \%$ saline was attenuated by preexposing the vanilla. The results support a Pavlovian analysis of the modification of salt preference through experience with the postingestive consequences of saline ingestion.
\end{abstract}

Thirst and sodium appetite are closely related in most animals, and behavior directed toward water and sodium is subject to a complex set of controls (e.g., Denton, 1982; Fitzsimons, 1979; Rolls \& Rolls, 1982). A number of investigators with varying orientations have suggested that both thirst and sodium appetite are modified by experience (Brookshire, 1967; Huston, 1975; Seligman, Mineka, \& Fillit, 1971; Toates, 1979; Weisinger, 1975). Perhaps the clearest and most dramatic example of the role of learning in saline preference is the formation of an aversion to the taste of saline as a result of pairing it with gastric distress (e.g., Parker \& Revusky, 1982). However, a more subtle demonstration of an acquired aversion to the taste of salt has been provided by Devenport (1973). In Devenport's study, rats preferred $.9 \%$ (isotonic) saline to water in an initial two-bottle test. However, after $24 \mathrm{~h}$ of exposure to either $.9 \%$ or $2 \%$ saline as the sole drinking fluid, this preference was reversed, and water was strongly preferred to isotonic saline.

Devenport's interpretation of these results invoked a learning mechanism. According to Devenport (see also Toates, 1979), during the conditioning treatment the taste of salt became associated with aversive postingestive consequences of prolonged drinking of isotonic or hypertonic saline solutions. The net result was a taste aversion, probably formed according to conventional principles of Pavlovian conditioning (e.g., Barker, Best, \& Domjan, 1977; Domjan, 1981; Logue, 1979). The idea that rats can learn

This research was supported by an operating grant from NSERC of Canada to V.M.L. and an I. W. Killam Postdoctoral Fellowship to J.M.K. The authors are indebted to Kent Berridge and Jeffrey Fairless for their comments on an earlier draft of the manuscript and to Andrew Delamater for help in conducting the experiments. Reprint requests should be sent to Vincent M. LoLordo at the Department of Psychology, Dalhousie University, Halifax, NS, Canada B3H $4 \mathrm{~J} 1$. an aversion to some stimulus that occurs in conjunction with relatively delayed postingestive consequences of sodium consumption is certainly consistent with the noted ability of a large number of postingestive consequences to induce taste aversions, even when minutes or hours separate experience of the taste and the consequences (e.g., Gamzu, Vincent, \& Boff, 1985; Riley \& Tuck, 1985).

The present set of experiments constitutes a replication and extension of Devenport's (1973) demonstration of the formation of an aversion to a taste associated with ingestion of saline. The original account of the phenomenon was cast in terms of associative learning, and certain predictions flow from that account. If this is a Pavlovian conditioning phenomenon, then certain standard Pavlovian phenomena, such as overshadowing and latent inhibition (Kehoe \& Gormezano, 1980; Mackintosh, 1974, 1976), should occur; the present study provided evidence of these phenomena. In addition, the present experiments explored the ability of some taste other than salt to acquire associative strength and elicit a conditioned response (CR) as a result of being paired with the postingestive consequences of the ingestion of $\mathrm{NaCl}$. The first experiment was an attempt to replicate Devenport's demonstration that the availability of isotonic saline as the sole drinking fluid for $24 \mathrm{~h}$ results in a reversal of the initial preference for isotonic saline over water.

\section{EXPERIMENT 1A}

\section{Method}

Subjects. Thirty-two albino male rats were obtained from Charles River Breeders, Canada, to serve as subjects. They were individually housed in a room with a 12:12 light-dark cycle in effect. They had continuous access to Purina Rodent Chow (5001). The first week in the laboratory served to habituate the rats to the light cycle and the food; for 2 days prior to the start of the experiment, they drank 
water ad lib from the graduated Nalgene tubes used to deliver liquid during the experiment.

Materials. Tap water was used for mixing solutions and as drinking water. The saline solution used in all two-bottle preference tests was $.9 \%(w / v) ~ N a C l$. A vanilla solution was prepared by mixing $2 \mathrm{ml}$ of Club House Pure Vanilla Extract (London, Canada) into 1 liter of solution to make a .2\% (v/v) solution. During conditioning, a saline-vanilla mixture was made by mixing $9 \mathrm{~g}$ of $\mathrm{NaCl}$ and $2 \mathrm{ml}$ of vanilla extract into 1 liter of solution.

Procedure. Following 2 days of access to ad-lib water in Nalgene tubes, the subjects were given their initial two-bottle pretest of preference between .9\% saline and water. This test was of 3 days' duration. For all subjects, the saline solution was in the left-hand bottle and the water was in the right-hand bottle. The subjects were then rank-ordered according to saline preference and assigned to groups so as to equalize initial mean preferences. There were 11 subjects in the saline (S) group, 11 in the water (W) group, and 10 in the saline-vanilla (SV) group.

A 24-h conditioning phase immediately followed the pretest. Each group of subjects had access to only one bottle of fluid during this phase. Group S had access to the $.9 \%$ saline solution, Group W had access to water, and Group SV had access to the saline-vanilla mixture.

A 48-h posttest immediately followed the conditioning phase and was conducted in the same manner as the pretest.

\section{Results}

Mean percent saline preferences in the two-bottle pretest were $41.9,40.4$, and 40.2 for Groups $S$, W, and SV, respectively. During conditioning, Group $S$ drank $65.3 \mathrm{ml}$ of saline solution, Group W drank $43.6 \mathrm{ml}$ of water, and Group SV drank $68.4 \mathrm{ml}$ of the saline-vanilla mixture [overall $F(2,30)=30.85, p<.001]$. Post hoc tests revealed that the two groups administered saline drank more than Group W ( $F=30.76)$, and that they did not differ from each other $(F<1)$.

The posttest saline preference scores did not indicate that the different conditioning experiences had any effect upon saline preference. The mean percent saline intake was 40.2 for Group S, 38.8 for Group W, and 26.8 for Group SV. Analysis of variance yielded a nonsignificant effect $[F(2,29)<1]$.

\section{Discussion}

Devenport (1973) and Wong (1977) observed a strong initial preference for isotonic saline over water in waterreared rats. This preference was not observed in the present experiment. Furthermore, a 24-h exposure to isotonic saline alone had no effect upon the preference for isotonic saline versus water in the present study, whereas the same manipulation produced a marked reversal of the preference over a 5-day measurement period in Devenport's experiment and a weaker reversal over a 2-day period in Wong's experiment.

The basis for these discrepancies is unknown. The rats used in the present study were fed standard Purina Chow, as were the rats used by Wong. Thus, the initial absence of a preference for isotonic saline cannot be attributed to a dietary regimen unique to this laboratory. Different strains of rats were used in the three studies: Wistar rats by Devenport, Long-Evans rats by Wong, and SpragueDawley rats in the present study. Although there was a difference in the magnitude of the effects observed by Devenport (1973) and Wong (1977), it seems unlikely that a strain difference is responsible for the complete absence of an effect observed here.

\section{EXPERIMENT 1B}

The first experiment in Devenport's (1973) series utilized a $2 \%$ saline solution in conditioning, and the subsequent reduction in preference for isotonic saline was slightly greater in magnitude in that case than when $.9 \%$ saline was used as the conditioning fluid. In Experiment 1B, Groups S and SV of Experiment $1 \mathrm{~A}$ were administered a $2 \%$ saline solution during the conditioning phase. In Pavlovian terminology, a stronger unconditioned stimulus (US), one that produces greater cellular dehydration (Rolls \& Rolls, 1982), was used.

\section{Method}

Subjects. The subjects of Experiment 1A retained their original group assignments.

Procedure. A conditioning phase was instituted immediately after the two-bottle posttest of Experiment 1A. During this 24-h phase, Group $\mathbf{S}$ had access to a $2 \%$ (w/v) solution of $\mathrm{NaCl}$, Group W had access to water, and Group SV had access to a mixture that was $2 \% \mathrm{NaCl}(\mathrm{w} / \mathrm{v})$ and $.2 \%$ vanilla $(\mathrm{v} / \mathrm{v})$. After this conditioning phase, all subjects were administered a 48-h two-bottle test of preference between $.9 \%$ saline and water. Finally, all subjects were administered a 24-h two-bottle test of preference between .2\% vanilla and water

\section{Results}

The data were analyzed by conducting separate analyses of variance (ANOVAs). After each ANOVA, a set of mutually orthogonal contrasts was constructed, as recommended by Rodger (1974, 1975). In all cases, Rodger's (1975) published F tables were consulted for these post hoc tests, and a decision-based rejection criterion of $\mathrm{p}<.05$ was adopted.

The groups differed in mean amount consumed during the 24-h conditioning phase [overall $F(2,27)=9.82$, $\mathrm{p}<.01]$. Group $\mathrm{W}$ drank less fluid $(42.7 \mathrm{ml})$ during the $24 \mathrm{~h}$ of conditioning than Group S $(69.4 \mathrm{ml})$ and Group SV $(61.0 \mathrm{ml})$ combined $(F=9.12)$, whereas the latter two groups drank equal amounts $(F<1)$.

Panel A of Figure 1 illustrates mean saline preference values for the three groups during the 48 -h posttest that followed the $2 \%$ saline conditioning phase. As inspection of the figure suggests, the groups differed in their saline preferences [overall $F(2,29)=4.45, p<.05$ ]. Post hoc orthogonal contrasts of the form recommended by Rodger $(1974,1975)$ indicated that Groups $S$ and W differed from each other $(F=4.15)$ and that Group SV's mean did not differ from the average of Groups $S$ and $W(F=.65)$. The logical implication of this decision set (Rodger, 1974) is that the true means are ordered $\mathrm{W}>\mathrm{SV}>\mathrm{S}$.

During the first $24 \mathrm{~h}$ of the two-bottle test of saline preference, Groups S and SV drank equal total amounts of fluid (saline + water; $F<1$ ). These two groups combined drank more total fluid than did Group W [overall 


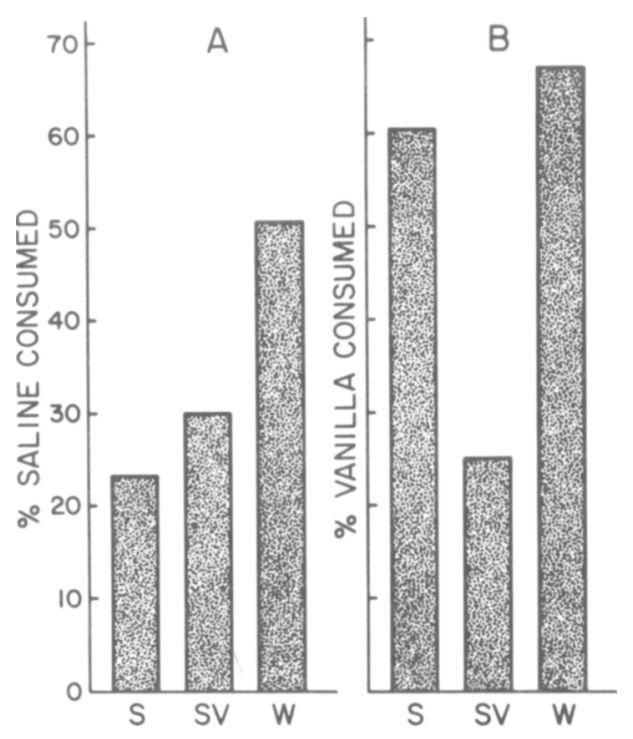

Figure 1. Posttest preference scores, Experiment 1B. Panel A: saline/(saline + water); Panel B: vanilla/(vanilla + water).

$\mathrm{F}(2,29)=8.12, \mathrm{p}<.01 ; \mathrm{F}$ for contrast $=7.62]$, suggesting that members of Groups S and SV experienced residual thirst following their conditioning experience with saline. However, on Day 2 of the posttest, the total fluid intake values were approximately equal for the three groups $[\mathrm{F}(2,29)=1.19, \mathrm{p}>.10]$.

Panel B of Figure 1 depicts the vanilla preference scores for the three groups. As inspection of Figure 1B suggests, Group SV drank a lower percentage of vanilla than did either Group S or Group W [overall $F(2,29)=11.01$, $\mathrm{p}<.01]$. Post hoc tests (Rodger, 1974) indicated that Groups $S$ and $W$ did not differ from each other $(F=.24)$, but that these groups combined differed from Group SV $(F=10.74)$.

\section{Discussion}

Allowing rats to drink only hypertonic saline for a day led to a generalized conditioned aversion to isotonic saline. The conditioning group, Group S, drank a significantly lower percentage of saline in the posttest than in the pretest, confirming Devenport's (1973) observations. In contrast, the control group, Group W, drank a nonsignificantly higher percentage of saline in the posttest than in the pretest. Since Group S drank a larger absolute amount of liquid than Group W during the posttest, one might wish to interpret these results as reflecting increased thirst and therefore increased water drinking in Group S, rather than as a true aversion to the taste of saline. This interpretation was ruled out by one of Devenport's (1973) experiments, in which the posttest was not administered immediately after conditioning but was delayed 7 days while the rats had continuous access to water. In spite of this delay, the saline aversion remained intact. However, such an aversion can be extinguished. Wong (1977) showed that the aversion to saline decreased over the course of a 5-day two-bottle posttest, during which the rats drank enough water to mitigate any negative postingestional consequences of the isotonic saline they consumed.

The novel finding of Experiment $1 \mathrm{~B}$ is that adding another taste to the saline solution during the conditioning phase can produce the outcomes predicted by a Pavlovian model of the phenomenon. First, an overshadowing effect was observed: the aversion to the saline taste was attenuated when saline had been presented concurrently with vanilla, another potential predictor of the $(2 \%)$ salineinduced discomfort. Second, the vanilla itself was avoided following its presentation during the conditioning phase. This demonstration provides support for an interpretation of Devenport's findings in terms of Pavlovian conditioning.

\section{EXPERIMENT 2}

The second experiment in essence replicates the procedure of Experiment 1B with naive rats, and extends the Pavlovian analysis of the phenomenon observed in that experiment by making an additional manipulation. When organisms have been exposed to nonreinforced presentations of a conditioned stimulus (CS) before the start of Pavlovian conditioning, they acquire the CR more slowly than nonpreexposed controls. This phenomenon, which is called latent inhibition (Lubow, 1973), has been observed in taste aversion learning (e.g., Best \& Barker, 1977; Domjan, 1972), as well as in many other procedures. Because Experiment 1B showed that the taste of vanilla could serve as a CS in the present paradigm, we used it in the preexposure manipulation of Experiment 2. Using vanilla as the target taste allowed us to preexpose the CS without preexposing the US, something that is not possible with a saline solution. In Experiment 2 a new treatment group, Group PxV, was exposed to vanilla prior to receiving the saline-vanilla mixture during the conditioning phase. If preexposure to vanilla reduces the conditionability of vanilla, then the ability of vanilla to overshadow or compete with the taste of saline as a predictor of the postingestional consequences of drinking hypertonic saline should be reduced, and the rats should acquire a stronger aversion to saline than rats in Group SV, which had no preexposure to vanilla (Carr, 1974). In the most extreme case, rats in Group PxV should exhibit a fullblown saline aversion, as though the vanilla had not been present during conditioning.

\section{Method}

Subjects. The subjects were 40 experimentally naive male albino rats. They were obtained, housed, and fed as in Experiment 1 .

Procedure. The subjects were randomly assigned to four groups of 10 subjects each. Three groups were treated as in Experiment 1 , and were named similarly: Group S2, Group SV2, and Group W2. The fourth group, Group PxV, was administered a vanilla solution, instead of water, as its sole drinking fluid for the 2 days prior to the initial two-bottle test. During these 2 days, Groups W2, S2, and SV2 drank water. In this preexposure phase and during conditioning, the concentration of vanilla flavoring was $.4 \%$, slightly higher than that in Experiment 1. 
After the 2-day preexposure phase, all subjects were administered the initial two-bottle choice between $.9 \%$ saline and water for $72 \mathrm{~h}$. A 24-h conditioning phase followed. During the conditioning phase, Group S2 had only $2 \%$ saline to drink and Group W2 had only water to drink. Group SV2 and Group PxV drank the mixture consisting of $2 \%$ saline and $.4 \%$ vanilla. Immediately following the conditioning phase, two-bottle choices between $.9 \%$ saline and water and between $.4 \%$ vanilla and water were administered, each lasting $48 \mathrm{~h}$. Half of the subjects in each group received the saline-water posttest first, followed by the vanilla-water posttest; the remaining subjects received the tests in the reverse order.

Immediately after the end of the posttest, all subjects were given continuous access to water and food for a week. Then the experiment was repeated, with minor modifications; this repetition of the procedures will be referred to as the second cycle. The vanilla preexposure phase of the second cycle lasted only $24 \mathrm{~h}$, followed immediately by a $24-\mathrm{h}$ conditioning phase, which was identical to the original conditioning. This conditioning phase was followed by twobottle posttests, as in the first part of the experiment. Conditioned effects should not be attenuated by repetition of the conditioning and testing procedures, whereas nonassociative processes such as neophobia should be diminished.

\section{Results}

Group PxV drank $45.6 \mathrm{ml}$ of the vanilla solution on the first day of preexposure and $47 \mathrm{ml}$ on the second day. Water consumption in Group PxV was $52.2 \mathrm{ml}$ on the day before preexposure, and vanilla intake was significantly lower than water intake (matched pairs $t$ test, $\mathrm{p}<.01$ ). When the procedures were repeated (second cycle), mean consumption of vanilla in the preexposure phase was $50.2 \mathrm{ml}$.

Once again, the pretest scores of saline preference averaged below $50 \%$. The means for the four groups are shown in the left-hand panel of Figure 2. The saline preference score for Group PxV appears to be lower than

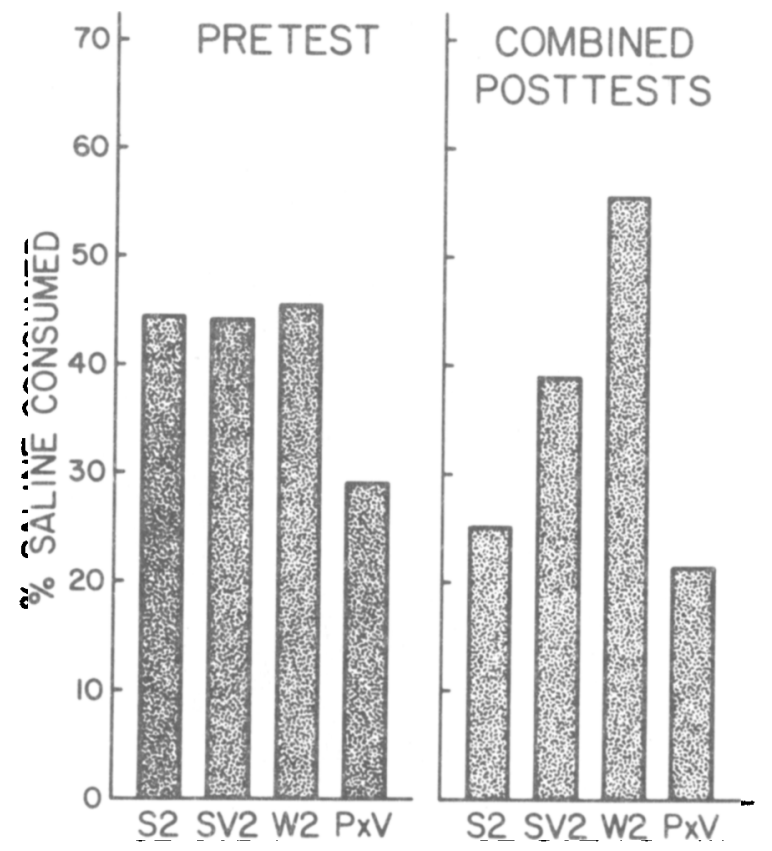

Figure 2. Pre- and posttest saline preference scores, Experiment 2.

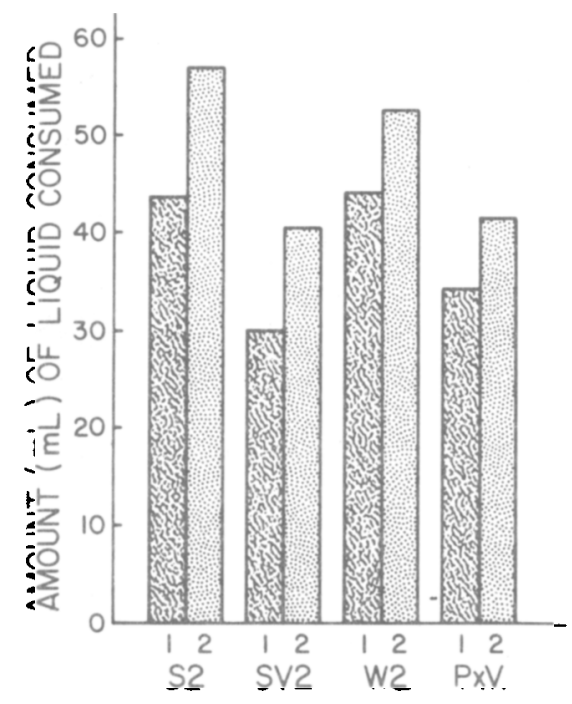

Figure 3. Total fluid consumption during conditioning phases of Cycles 1 and 2, Experiment 2. Group SV2 and Group PxV drank the saline-vanilla mixture, Group $\$ 2$ drank saline solution, and Group W2 drank water.

those of the other three groups, but the effect of groups at the pretest was not significant $[\mathrm{F}(3,72)=2.62$, $.05<\mathrm{p}<.10]$.

Figure 3 shows consumption during the conditioning phases. An ANOVA indicated that there was a groups effect [overall $F(3,36)=4.66, p<.01]$ during the conditioning period of the first cycle. The two groups exposed to the vanilla-saline mixture drank similar amounts of fluid $(F<1)$, and both of these groups drank less than Group S2 and Group W2 $[\mathrm{F}(3,36)=4.35, \mathrm{p}<.05$; Rodger, 1975]. Groups S2 and W2 did not differ from each other $(F<1)$. The patterns of consumption during the conditioning phase of the second cycle were similar to those of the first, but the difference between groups only approached statistical significance [overall $\mathrm{F}(3,34)$ $=2.72, .05<\mathrm{p}<.10$ ].

The preference data from the saline-water posttests of the two cycles were very similar to each other. The righthand panel of Figure 2 presents the combined results of these two tests. Because the initial saline preference of Group PxV was marginally lower than that of the other three groups, the one pretest and two posttest preference scores of all subjects were included in a repeated measures ANOVA. There was a significant groups $\times$ tests interaction $[F(6,72)=5.45, p<.01]$, and this was further analyzed by testing each group for changes in preference over tests (Kirk, 1968). Group W2 exhibited a significant increase in saline preference from the pretest to posttests $[F(2,72)=8.8, p<.01]$. The saline preference of Group S2 decreased significantly $(F=11.57$, $\mathrm{p}<.01)$.

The saline preference scores of Groups SV2 and PxV exhibited numerical, but not significant, decreases from pretest to posttests ( $F s=2.22$ and 1.69 , respectively, ps $>$.10). The aversion exhibited by Group SV2 was at- 
tenuated relative to that of Group $\mathrm{S} 2$, as expected. The results for Group PxV were somewhat ambiguous, because the rats in that group tended to have lower saline preference scores in pretest than the other groups, although not significantly lower. In some respects, however, the changes in saline preference in Group PxV are consistent with the Pavlovian conditioning hypothesis. If only rats with pretest preference scores in the middle of the distribution, that is, from $25 \%$ to $50 \%$, are considered, there were 4-6 rats in each of the various groups. All 4 rats in Group W2 showed an increase in saline preference as a result of conditioning; 5 of 6 rats in each of Groups S2 and PxV showed a decrease, and 1 an increase; only 2 rats in Group SV2 showed a decrease, and 3 showed an increase. Thus, the group preexposed to vanilla prior to conditioning to the saline-vanilla compound behaved like the group conditioned to saline alone, rather than like the group given no preexposure prior to compound conditioning.

The combined results of the vanilla-water tests of the two cycles are shown in Figure 4. Inspection of the figure suggests that Group W2 had the highest vanilla preference score, whereas the scores of Groups S2 and PxV were somewhat lower. Group SV2 exhibited the least acceptance of vanilla. An ANOVA revealed a significant effect of groups [overall $\mathrm{F}(3,36)=10.12$, $\mathrm{p}<.001$ ] but a nonsignificant groups $\times$ tests interaction $[\mathrm{F}(3,36)=2.6, \mathrm{p}>.05]$. Post hoc tests (Rodger, 1974, 1975 ) indicated that Groups W2 and SV2 differed from each other $(F=9.63)$. Groups $S 2$ and PxV did not differ from each other $(F<1)$, and their combined scores did not differ from the combined scores of the extreme groups $(\mathrm{F}<1)$. These results logically imply that the group means were ordered $\mathrm{W} 2>\mathrm{S} 2=\mathrm{PxV}>\mathrm{SV} 2$.

\section{Discussion}

As in Experiment $1 \mathrm{~B}$, rats given only $2 \%$ saline solution to drink during conditioning showed a decreased acceptance of $.9 \%$ saline in the posttest, relative to their

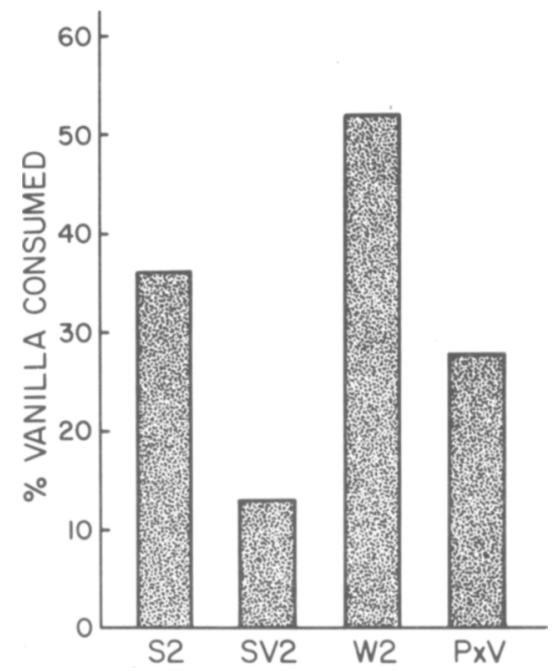

Figure 4. Vanilla preference scores, Experiment 2. initial preference and relative to that of Group W2. It is clear that Group SV2 did not acquire as strong an aversion to the saline as did Group S2, again replicating the results of Experiment 1B.

The Pavlovian conditioning hypothesis stated that rats in Group PxV would acquire a robust aversion to saline, but not to vanilla, to which they had been previously exposed. Interpretation of Group PxV's response to saline is problematic. Perhaps because this group initially had a low saline preference (although not significantly lower than that of the other groups), its decrease in saline preference from pretest to posttest was not statistically significant. However, 8 of 10 rats in this group did show a decrease in saline preference, and this change should be viewed in the context of an increase in preference shown by rats in Group W2. Moreover, Group PxV's mean preference score was the lowest of all groups in the posttest. Thus, in spite of their initially low saline preference scores, it is reasonable to assert that the rats in Group PxV did learn an aversion to saline.

The results of the vanilla preference tests suggest that preexposing the vanilla attenuated the aversion to it. In fact, Group PxV was no more averse to the taste of vanilla than was Group S2, which did not experience vanilla during conditioning. The aversion to vanilla exhibited by Group S2 relative to Group W2 must be attributed to stimulus generalization from the taste of salt. Group SV2, as expected, acquired a stronger aversion to the taste of vanilla than did the other groups.

In accord with the Pavlovian conditioning hypothesis, Group PxV was more accepting of vanilla than was Group SV2 following conditioning, even though the two groups drank equivalent amounts of the saline-vanilla mixture during conditioning. These data suggest that the ability of vanilla both to enter into associations with the US and to compete with saline for association with the US was attenuated by nonreinforced exposures to vanilla.

\section{GENERAL DISCUSSION}

The overall pattern of results attests to the generality of Devenport's demonstration of salt-preference modification. However, there were certain differences between the present observations and previous ones. The subjects of the present study did not initially prefer .9\% saline to water to the extent noted in previous reports. Moreover, the present study constituted a failure to obtain a reduction in preference following conditioning with $.9 \%$ saline.

Conditioning with a $2 \%$ saline solution did result in a generalized aversion to the $.9 \%$ saline, and this outcome is certainly similar to the original effect reported by Devenport (1973). Two major points regarding this effect merit discussion. The first is that the phenomenon is the result of Pavlovian conditioning, and can be affected by such Pavlovian procedures as overshadowing and latent inhibition.

The SV groups of both experiments exhibited a more modest aversion to the taste of saline than the $S$ groups, even though they experienced the taste of saline in con- 
junction with its postingestive consequences during the conditioning phase. This is a demonstration of the wellknown overshadowing phenomenon (Kehoe \& Gormezano, 1980; Mackintosh, 1974, 1976). Like other demonstrations of overshadowing, this one can be subjected to the criticism that the reduced aversion to saline exhibited by the SV groups was due merely to greater generalization decrement between the conditions of conditioning and those of test. However, the SV groups' acquisition of an aversion to vanilla, an arbitrarily chosen flavor paired with prolonged drinking of hypertonic saline, adds support for the assertion that the observed overshadowing reflects competition between the tastes of saline and vanilla for the acquisition of associative strength. Additionally, this finding argues against any privileged role for the taste of salt as a predictor of its own postingestive consequences. In the present case, vanilla seems to have acquired control over behavior roughly as readily as saline. In another illustration of this point, Fudim (1978) reported that rats rendered sodium-deficient exhibited a preference for a flavor which had previously been associated with the taste of salt. Thus, there is evidence from both appetitive and aversive preparations that flavors associated with salt can be treated as salt surrogates by rats.

Consumption of liquid during the conditioning phases did not yield any indication of an aversion to the taste of saline solution, in accord with Devenport's (1973) findings. As in previous studies, the subjects in Groups $\mathrm{S}$ and SV of Experiment 1A drank more .9\% saline solution during conditioning than the $\mathrm{W}$ group drank water. Also consistent with Devenport's findings, rats drank at least as much $2 \%$ saline solution as water during the other conditioning phases.

The nature of the effective unconditioned response to prolonged consumption of hypertonic saline merits discussion. Devenport (1973) hypothesized that the US in the present preparation was thirst resulting from cellular dehydration. It is difficult to assert that there was no aversive postingestive consequence of salt ingestion other than thirst. However, Oatley and Toates (1973) studied the effects of acute hypertonic saline injections on eating in the rat. Hypertonic saline injections dehydrate the intracellular volume. Such injections were found to inhibit eating during a period starting $.5 \mathrm{~h}$ after the injection and continuing for $1 \mathrm{~h}$ if no water accompanied the food. However, if water was made available along with the food in this preparation, normal amounts of food were eaten. Thus, at least in this acute preparation, the effect of salt was to inhibit eating as a result of a readily reversible water deficit. The effect of salt in the experiments of Oatley and Toates (1973) did not seem to be a general disruption of the organism's behavior. With regard to the present experiments, casual observation of the animals suggested that they were not experiencing distress during the conditioning phase. Presumably in the present experiments, as in those of Oatley and Toates (1973), excess saline resulted in thirst. The temporarily high posttest fluid intake of saline- trained animals suggests that they were compensating for a water deficit, which seems to have been eliminated by the second day of two-bottle testing.

The ability of thirst to serve as a US in a Pavlovian conditioning preparation has been a subject of enquiry for some time (e.g., Huston, 1975; Weisinger, 1975). If adequate documentation for a role of naturally induced thirst can be produced, the domain of conditioning theory will be expanded. So far, most preparations have used acute thirst, induced by injections and having a rapid time course, to induce conditioned drinking rather than a taste aversion. The conditions that might favor conditioned drinking over the formation of a taste aversion are unclear at present. Conditioned taste aversions are commonly induced with acute postingestive consequences, so the time-course of the US does not appear to be critical. One might speculate that edible flavored substances that predict thirst would be avoided, whereas external stimuli, such as the white box used as a CS by Mineka and Seligman (1975), would control conditioned drinking of water. Mineka and Seligman mentioned that they were unable to condition thirst using mere water deprivation as a US. In any event, if the original interpretation of the saline preference shift (Devenport, 1973) is correct, then the preparation can profitably be used to study classical conditioning of thirst using a less acute, and possibly more "natural," US.

\section{REFERENCES}

Barker, L., Best, M. R., \& Domuan, M. (Eds.) (1977). Learning mechanisms in food selection. Waco, TX: Baylor University Press. BEST, M. R. \& BARKER, L. (1977). The nature of "learned safety" and its role in the delay of reinforcement gradient. In L. M. Barker, M. R. Best, \& M. Domjan (Eds.), Learning mechanisms in food selection. Waco, TX: Baylor University Press.

Brookshire, K. H. (1967). Reinforcement values of water and hypotonic saline in discrete-trial situations. Journal of Comparative \& Physiological Psychology, 63, 145-148.

CARR, A. F. (1974). Latent inhibition and overshadowing in conditioned emotional response conditioning with rats. Journal of Comparative \& Physiological Psychology, 86, 718-723.

Denton, D. (1982). The hunger for salt. New York: Springer-Verlag. DevenPort, L. D. (1973). Aversion to a palatable saline solution in rats: Interactions of physiology and experience. Journal of Comparative \& Physiological Psychology, 83, 98-105.

DomJAN, M. (1972). CS preexposure in taste-aversion learning: Effects of deprivation and preexposure duration. Learning \& Motivation, 3 , 389-402.

DomJaN, M. (1981). Ingestional aversion learning: Unique and general processes. In J. S. Rosenblatt, R. A. Hinde, C. Beer, \& M.-C. Busnel (Eds.), Advances in the study of behavior (Vol. 11). New York: Academic Press.

Fitzsimons, T. J. (1979). The physiology of thirst and sodium appetite. Monographs of the Physiological Society (No. 35). Cambridge: Cambridge University Press.

FUDIM, O. (1978). Sensory preconditioning of flavors with a formalinproduced sodium need. Journal of Experimental Psychology: Animal Behavior Processes, 4, 276-285.

Gamzu, E., Vincent, G., \& Boff, E. (1985). A pharmacological perspective of drugs used in establishing conditioned food aversions. In N. Braveman \& P. Bronstein (Eds.), Experimental assessments and clinical applications of conditioned taste aversions. New York: New York Academy of Sciences. 
Huston, I. P. (1975). Classical conditioning of consumatory behavior. In G. Peters, T. J. Fitzsimons, \& L. Peters-Haefeli (Eds.), Control mechanisms of drinking. New York: Springer-Verlag.

KehOE, E. J., \& Gormezano, I. (1980). Configuration and combination laws in conditioning with compound stimuli. Psychological Bulletin, 87, 351-378.

KIRK, R. E. (1968). Experimental design: Procedures for the behavioral sciences. Monterey, CA: Brooks/Cole.

LOGUE, A. (1979). Taste aversion and the generality of the laws of learning. Psychological Bulletin, 86, 276-296.

Lubow, R. E. (1973). Latent inhibition. Psychological Bulletin, 79, 398-407.

Mackintosh, N. J. (1974). The psychology of animal learning. New York: Academic Press.

Mackintosh, N. J. (1976). Overshadowing and stimulus intensity. Animal Learning \& Behavior, 4, 186-192.

Mineka, S., \& Seligman, M. E. P. (1975). Conditioned drinking as avoidance learning. Journal of Comparative \& Physiological Psychology, 88, 69-80.

OATLEY, K., \& TOATES, F. M. (1973). Osmotic inhibition of eating as a subtractive process. Journal of Comparative \& Physiological Psychology, 82, 268-277.

Parker, L. A., \& Revusky, S. (1982). Generalized conditioned flavor aversions: Effects of toxicosis training with one flavor on the preference for different novel flavors. Animal Learning \& Behavior, 10, 505-510.
RILEY, A. L., \& TUCK, D. L. (1985). Conditioned taste aversions: A bibliography. In N. Bravernan \& P. Bronstein (Eds.), Experimental assessments and clinical applications of conditioned taste aversions. New York: New York Academy of Sciences.

RoDGER, R. S. (1974). Multiple contrasts, factors, error rate and power. British Journal of Mathematical \& Statistical Psychology, 27, 179-198.

RODGER, R. S. (1975). The number of non-zero, post hoc contrasts from ANOVA and error-rate. British Joumal of Mathematical \& Statistical Psychology, 28, 71-78.

Rolls, B. J., \& Rolss, E. T. (1982). Thirst. Cambridge: Cambridge University Press.

Seligman, M. E. P., Mineka, S., \& Fillit, H. (1971). Conditioned drinking produced by procaine, $\mathrm{NaCl}$, and angiotensin. Journal of Comparative \& Physiological Psychology, 77, 110-121.

TOATES, F. M. (1979). Homeostasis and drinking. Behavioral \& Brain Sciences, 2, 95-136.

WEISINGER, R. S. (1975). Conditioned and pseudoconditioned thirst and sodium appetite. In G. Peters, T. J. Fitzsimons, \& L. Peters-Haefeli (Eds.), Control mechanisms of drinking. New York: Springer-Verlag. WoNG, R. (1977). Adaptation to long-term saline consumption and later saline preference. Behavioral Biology, 19, 389-393.

(Manuscript received May 28, 1985; revision accepted for publication October 18, 1985.) 